East African Medical Journal Vol. 87 No. 1 January 2010

TEMPOROMANDIBULAR JOINT DISLOCATION IN NAIROBI

L.K. Sang, BDS (Nbi) Dental Officer, Kajiado District Hospital, P.O. Box 16949-00620 Nairobi, Kenya, E. Mulupi, BDS (Nbi) Dental Officer, Moi Teaching and Referral Hospital, P.O. Box 387-50103, Malava, Kenya, M.K. Akama, BDS, MDS (Nbi) Lecturer, Department of Oral and Maxillofacial Surgery, Oral Medicine and Oral Pathology, Faculty of Dental Sciences, J.M. Muriithi, BDS, Senior Lecturer, Department of Surgery, Faculty of Medicine, F. G. Macigo, BDS, MPH, PGD-STI's (Nbi), Senior Lecturer, Department of Periodontology and Community Dentistry, Faculty of Dental Sciences and M.L. Chindia, BDS (Nbi), MSc Med. (Lond), FFDRSC (Ireland), Associate Professor, Department of Oral and Maxillofacial Surgery, Oral Medicine and Oral Pathology, Faculty of Dental Sciences, University of Nairobi, P.O. Box 19676-00202, Nairobi, Kenya

Request for reprints to: Dr. L. K. Sang, Kajiado District Hospital, P.O. Box 16949-00620, Nairobi, Kenya

\title{
TEMPOROMANDIBULAR JOINT DISLOCATION IN NAIROBI
}

\author{
L. K. SANG, E. MULUPI, M. K. AKAMA, J. M. MURIITHI, F. G. MACIGO and M. L. CHINDIA
}

\begin{abstract}
Background: Despite the diverse conservative and surgical modalities for the management of temporomandibular joint (TMJ) dislocation and the controversy that surrounds them, very little has been done within the East-African setup in terms of highlighting and provoking greater interest in the epidemiology and management of TMJ dislocation.

Objective: To audit the pattern of occurrence, demographics, aetiology and enumerate the treatment modalities of TMJ dislocation at the oral and maxillofacial surgery division (OMFS) of the University of Nairobi Dental Hospital.

Design: Descriptive cross-sectional study.

Setting: University of Nairobi Dental Hospital (UNDH) from January 1995 to July 2005.

Results: Twenty nine patients had been diagnosed and managed for TMJ dislocation. Twenty $(69 \%)$ were females and nine $(31 \%)$ were males. Their ages ranged from 10-95 years with a mean of 42 years. The cases managed were primarily chronic in nature. The most common form being anterior TMJ dislocation, accounting for twenty-five $(86.2 \%)$ cases. Trauma was implicated as an aetiology in only five $(\mathbf{1 7} \%)$ of the cases while the remaining majority of twenty four $(83 \%)$ cases were spontaneous. Amongst the causes of spontaneous TMJ dislocation, yawning was the most common accounting for fourteen cases $(48.3 \%$ ). Dislocations caused by trauma were found to be $\mathbf{1 2 . 6}$ times more likely to be associated with other injuries than spontaneous dislocations. Anterior TMJ dislocations were found to be 1.3 times more likely to be associated with absence of molars than posterior TMJ dislocations. Anatomical aberrations, as predisposing factors, were not a significant finding in this research. Eight $(28 \%)$ of the cases were managed conservatively. Twenty one $(72 \%)$ of the cases were managed surgically. The eminectomy was the most common technique with a $75 \%$ success rate. The highest incidence of TMJ dislocation occurs in the $3 \mathrm{rd}-5$ th decade with a female preponderance with bilateral anterior TMJ dislocation being the most common. Most of the cases were managed surgically with eminectomy being the preferred technique with the highest success rate. A study needs to be undertaken to determine reasons' why conservative modalities are least employed in the management of TMJ dislocation in our setup and what can be done about it.
\end{abstract}

\section{INTRODUCTION}

A dislocation is a displacement of one component of thejoint beyond its normal limitswithout spontaneous return to its normal position. It results when the condylarhead is displaced out of the glenoid fossa, but still remains within the capsule. TMJ dislocation can be classified as anterior, posterior, lateral and superior based on the direction of displacement and location of the condylar head. Anterior TMJ dislocation is the most common and is more frequent in females. It is rare in children and elderly people than in the prime of life. The other variants of TMJ dislocation are exceptionally rare and almost exclusively associated with trauma by 1983 only 19 cases of superior TMJ dislocation had been reported in literature (1). 
TMJ dislocation represents 3\% of all articular body dislocations (2). Spontaneous dislocation of the TMJ occurs in up to $5.8 \%$ of people during their lifetime and is most commonly seen in those in the 2nd and 3rd decades of life (3). Similar to other TMJ afflictions, the highest incidence of TMJ dislocation is reported in females, even though the reasons for this fact are still not fully understood (4-6) the reverse is the case in other studies $(1,8,9)$.

Most cases of dislocation occur spontaneously during voluntary wide opening of the mouth such as while yawning, laughing, during protracted dental procedures or during a seizure (10). Trauma to the maxillomandibular complex is also a common cause of dislocation. According to published statistics, $44-99 \%$ of TMJ problems are caused by trauma (11). Forced passive opening of the mouth such as during laryngoscopy, bronchoscopy and intubation in patients, with or without hypermobility of the TMJ, may also lead to dislocation $(12,13)$. Yawning during induction of anaesthesia is frequently seen with propofol and in the stage of sedation, forceful yawning may occur (14). TMJ dislocation has been reported to occur after oral airway, nasogastric tube placement and laryngeal mask airway insertion with the jaw thrust technique in an intubated patient (15). Insertion of a Boyle-Davis mouth gag during tonsillectomy can lead to dislocation of the TMJ or pain as a result of postoperative joint dysfunction (16). The clinical impression is that temporary dislocation of the TMJ occasionally occurs during tonsillectomy (17). TMJ dislocation has been reported with both epilepsy and dystonia caused by neuroleptic medication (phenothiazines e.g compazine) and certain antiemetics (e.g metoclopramide) that produce extrapyramidal effects (18).

Predisposing factorsfor TMJ dislocation include, shallow mandibular fossae, atrophic eminence, underdeveloped condyles, occlusal disharmony, internal derangements of the TMJ and capsular ligaments, neurological disorders and hypermobility associated with systemic disorders (10).

Dislocations of the TMJ may be further classified as acute, chronic and recurrent (Habitual/ Neurogenic). The management of acute dislocation of the TMJ has not changed significantly over the years. Manual reduction assisted by sedation is what is commonly used. Other techniques include spontaneous reduction described by Johnson (19) based upon the theory that dislocation is maintained by muscle spasms and treatment consists of injection of the joint with lignocaine hydrochloride that induces spontaneous reduction after approximately one minute. According to Awang (20), induction of a gag reflex by probing the soft palate results in reflex neuromuscular activity that causes reduction.

Recurrent dislocation is uncommon. Boering found an incidence of $1.8 \%$ in a population of 400 patients with symptomatic TMJ disorders (21).
Conservative management involves a careful search and elimination of the predisposing factors occasioning muscle spasm and extrapyramidal effects. This is followed by maxillomandibular fixation to limit translatory movements (22). Other conservative modalities include sclerotherapy, documented success rates range from 72 to $75 \%$. Multiple treatments are often necessary and the treatment is considered temporary or reversible $(23,24)$. The chemical denervation of the lateral pterygoid muscle using botulinum toxin injections is another therapeutic alternative, thus restricting the angle of mouth opening to prevent dislocation (25). Surgery should be reserved for those cases which fail to respond to conservative therapy (1).

Rowe and Caldwell (26) reviewed literature and found that chronic dislocation were not rare.Chronic dislocation of the mandibleusually occurs when a case of acute dislocation is left untreated or is inadequately managed. Hayward stated that the longer the mandible remains dislocated the more difficult the management of the condition because of more severe muscle spasm and fibrotic periarticular changes; he however points out that conservative modalities including occlusal splints, physiotherapy, traction, sclerosants and orthodontics may often succeed in cases of prolonged dislocation of the TMJ (27). Fordyce (28) reviewed literature on chronic dislocation and pointed out that manual reduction, although generally difficult after one month from the time of dislocation, can be successfully accomplished after as long a period as six months. However, Gottlieb (29) found that only three of 24 long-standing cases were successfully reduced by manual manipulation, the others being reduced surgically. Another review reported that four out of 24 cases of long-standing dislocation were successfully reduced manually (7).

Various techniques have been proposed for the surgical management of TMJ dislocation and can be divided into two categories:

Operations that limit the condylar path (23).

(i) Arch down fracturing technique: This is one of the most common techniques and has a documented success rate of $91 \%$. The procedure does not violate the joint space and allows immediate, normal anterior movement with little limitation in opening. However it incurs risk of fracture and resorption of the distal segment.

(ii) Eminence augmentation techniques: Relies on a variety of bone grafts or plates to limit condylar translation.

(iii) Myotomy techniques: Attempts to disable the lateral pterygoid muscle allowing only rotational movement of the condyle. Its disadvantages include difficulty in visualisation, high risk of haemorrhage and muscle tissue may reattach during healing placing the long-term efficacy of the procedure in doubt. 
(iv) Condylectomy: Aims to achieve pseudoarthrosis (or nearthrosis) thus limiting range of mandibular motion.

Operations that enhance the condylar path (23).

(i) Eminectomy: This is one of the most common techniques and has a documented success rate of $95 \%$. It is recommended for patients who habitually dislocate and have a disability that inhibits controlled muscular movements.

(ii) Closed condylotomy: $81 \%$ success rate has been reported using this procedure however its usefulness has been limited by the potential for serious bleeding from the internal maxillary artery.

(iii) Meniscectomy

Despite the diverse conservative and surgical modalities for the management of TMJ dislocation and the controversy that surrounds them, very little has been done within the East-African setup in terms of highlighting and provoking greater interest in the epidemiology and management of TMJ dislocation. The purpose of this study was to compile a ten year retrospective audit of the characteristics of TMJ dislocation in our unit and document the demographics, etiology, risk factors and simply enumerate the treatment modalities. The findings of this study will serve as baseline data on TMJ dislocation within our setup.

\section{MATERIALS AND METHODS}

This study was approved by the Ethics, Research and Standards Committee of the Kenyatta National Hospital (KNH) and UNDH (Approval numberKNH$\mathrm{ERC} / 01 / 3050)$. A retrospective audit of patients who had been managed for TMJ dislocation from January 1995 to July 2005 was then accomplished by manually assessing the medical registry at the UNDH. Records of patients diagnosed with TMJ dislocation during this period were retrieved from the registry and information regarding age, sex, diagnosis, aetiology of dislocation, predisposing factors, associated injuries and treatment modalities offered, analysed. Analysis was done using the statistical software SPSS and significant differences were inferred at P-values less than 0.05 .

\section{RESULTS}

Socio-demographic information: Twenty nine patients had been diagnosed and managed for TMJ dislocation. Twenty $(69 \%)$ of the patients were female while nine $(31 \%)$ were male. Seventeen of the patients were married while twelve were either single or widowed.

The patients ranged in age from 10 to 95 years, with a mean of approximately 42 years. The p-values, using the turkey HSD post hoc tests for multiple comparisons, showed that TMJ dislocations were distributed amongst three main age sets and the highest frequency occurred in individuals between 31-50 years $(37.9 \%)$.

Cases of anterior TMJ dislocation were the most prevalent, involving eighteen female and seven male patients. Posterior TMJ dislocation was also encountered, involving two female and two male patients. No statistically significant relationship was found between the distribution of TMJ dislocation and gender implying a random distribution $(\mathrm{P}>0.05)$.

PatternofTMJdislocations andaetiologicfactors: Bilateral anterior TMJ dislocation was noted to be the most common accounting for twenty two $(76 \%)$ of the cases, involving sixteen (55\%) female and six (21\%) male patients. Unilateral anterior TMJ dislocation accounted for only three (10\%) cases involving one $(3 \%)$ male and two $(7 \%)$ female patients. Three $(10.5 \%)$ cases of bilateral posterior TMJ dislocation were documented involving one $(3.5 \%)$ female and two $(7 \%)$ male patients. Unilateral posterior TMJ dislocation accounted for one (3.5\%) case involving a female patient (Table 1).

Table 1

Pattern of TMJ dislocation as distributed by gender

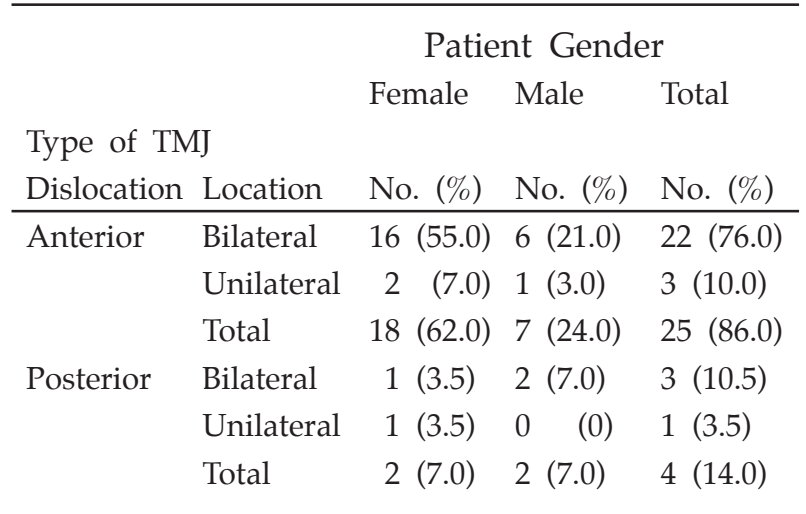

Trauma was implicated as an aetiology in only five $(17 \%)$ of the documented cases. The majority of cases, that is twenty-four $(83 \%)$, were all spontaneous in nature. Yawning was the most common cause of spontaneous dislocation accounting for fourteen $(48.3 \%)$ cases followed by laughing, eight $(27.6 \%)$ cases (Table 2). 
Table 2

Aetiologic factors associated with the TMJ dislocation

\begin{tabular}{lllr}
\hline & Aetiologic Factors & \multicolumn{2}{l}{ No. $(\%)$} \\
\hline Trauma & Accidents & 5 & 17.2 \\
Spontaneous & Yawning & 14 & 48.3 \\
& Laughing & 8 & 27.6 \\
& Abnormal occlusion & 1 & 3.4 \\
& Protracted & & \\
& Dental procedure & 1 & 3.4 \\
\hline Total & & 29 & 100
\end{tabular}

More female patients, that is eighteen $(75 \%)$, suffered spontaneous TMJ dislocation as compared to the six $(25 \%)$ male patients; a ratio of $1: 3$. Chi square tests revealed that females were 2.65 times likely to suffer spontaneous anterior TMJ dislocation than males, however there was no statistically significant relationship implying a random distribution $(\mathrm{P}>0.05)$.

Diagnostic Aids: Seventeen $(58.6 \%)$ of the cases were diagnosed with the aid of both TMJ tomograms and an orthopantomogram (OPG). Eight (27.6\%) and four $(13.8 \%)$ of the cases were diagnosed with the aid of either an OPG or TMJ tomograms respectively.

Pattern of TMJ dislocations and associated injuries: Out of the four cases of posterior TMJ dislocation three were associated with other injuries these being fractures, loss of teeth, intrusions, extrusions and lacerations. However, only one case of anterior TMJ dislocation was associated with other injuries. A statistically significant correlation was found between the pattern of TMJ dislocation and associated injuries and that posterior TMJ dislocations were 14.8 times more likely to be associated with other injuries than anterior TMJ dislocations $(\mathrm{P}<0.05)$.

Aetiologic factors and associated injuries: Three of the TMJ dislocations caused by trauma were associated with other injuries while only one of the spontaneous TMJ dislocations was associated with other injuries. An evaluation of the correlation between aetiologic factors and associated injuries found that dislocations caused by trauma are 12.6 times more likely to be associated with other injuries than spontaneous dislocations $(\mathrm{P}<0.05)$.

Pattern of TMJ dislocation and predisposing factors: Four of the cases of anterior TMJ dislocation were associated predisposing factors the most common being absence of molars. None of the cases of posterior TMJ dislocation were associated with predisposing factors (Table 3). Nostatistically significant correlation was found between the pattern of dislocation and the predisposing factors $(p>0.05)$. However, anterior TMJ dislocations were found to be 1.3 times likely to be associated with absence of molars and 0.3 times likely to be associated with other predisposing factors than posterior TMJ dislocations.

Table 3

Predisposing factors associated with each type of TMJ dislocation

Predisposing factor

Type of TMJ dislocation

\begin{tabular}{|c|c|c|c|c|c|}
\hline & & No. & $(\%)$ & No & $(\%)$ \\
\hline \multirow[t]{3}{*}{ Absence of molars } & Present & 3 & 10.3 & 0 & 0 \\
\hline & Edentulous & 1 & 3.4 & 0 & 0 \\
\hline & Absent & 21 & 72.4 & 4 & 13.8 \\
\hline \multirow[t]{2}{*}{ Occlusal disharmony } & Present & 0 & 0 & 0 & 0 \\
\hline & Absent & 25 & 86.2 & 4 & 13.8 \\
\hline \multirow[t]{2}{*}{ Shallow fossae } & Present & 1 & 3.4 & 0 & 0 \\
\hline & Absent & 24 & 82.8 & 4 & 13.8 \\
\hline \multirow[t]{2}{*}{ Atrophic eminence } & Present & 0 & 0 & 0 & 0 \\
\hline & Absent & 25 & 86.2 & 4 & 13.8 \\
\hline \multirow[t]{2}{*}{ Lax ligaments } & Present & 1 & 3.4 & 0 & 0 \\
\hline & Absent & 24 & 82.8 & 4 & 13.8 \\
\hline
\end{tabular}


Management of TMJ dislocations: Majority of the cases of TMJ dislocation that were managed were primarily chronic in nature. Manual reduction under general anaesthesia was the only conservative modality employed. Fifteen (51\%) of the cases of TMJ dislocation were managed conservatively with a $53 \%$ success rate there is eight (53\%) were successful and seven $(47 \%)$ unsuccessful. The unsuccessful cases were later managed surgically and were included in the surgical tally.

Twenty one $(72.4 \%)$ of the TMJ dislocation cases were managed surgically, sixteen $(76 \%)$ by eminectomy and five $(24 \%)$ by condylectomy. The cases managed surgically that were associated with postoperative complications, trismus and warranted other modalities to be instituted were termed as having had a poor outcome. The cases that were associated with minimal or no postoperative complications and increasing functionality were termed as having had a satisfactory outcome. Fourteen $(67 \%)$ of the cases that were managed surgically had a satisfactory outcome while seven $(33 \%)$ had a poor outcome. The eminectomy had a $75 \%$ success rate with twelve satisfactory and four poor outcomes. The condylectomy had a $40 \%$ success rate with two satisfactory and three poor outcomes.

\section{DISCUSSION}

Although there are several reports in literature on TMJ dislocation none have emanated from the East African region for considerable comparison.

In this study the highest incidence of TMJ dislocation was documented in individuals in the $3^{\text {rd }}$ to $5^{\text {th }}$ decade, this compares favourably with other studies (1). A higher female prevalence of $69 \%$ was also noted in this study similar to findings in previous studies (1,4-6) and this was statistically significant. Chisquare tests revealed that females were2.65times likely to suffer spontaneous anterior TMJ dislocation than males. Reasons for the vulnerability of females are not clear (4). The most common investigative modalities employed in the diagnosis of TMJ dislocation were a combination of OPG and TMJ tomograms probably because they are relatively inexpensive.

Similar to other findings $(2,23,24,30,31)$ most of the dislocations documented were anterior TMJ dislocations $(86 \%)$. Posterior TMJ dislocation, despite being a rare form of dislocation, accounted for $14 \%$ of the documented cases. Superior and lateral TMJ dislocations were not encountered in this series confirming their rarity as very few have been reported in literature (30). Previousstudies $(2,31)$ have revealed a majority of dislocations to be bilateral a finding similar to the current study. All this is partly cause related since majority of the dislocations occurred spontaneously $(83 \%)$ mainly during yawning $(48.3 \%)$ and laughing $(27.6 \%)$ while trauma was implicated in $17 \%$ of the cases.
Anterior TMJ dislocations were found to be less likely to have been associated with other injuries as opposed to posterior dislocations. This, as mentioned earlier, is also cause-related and may be explained by the fact that in the current study dislocations caused by trauma are 12.6 times more likely to be associated with other injuries than spontaneous dislocations, a finding echoed in other studies $(24,30,31)$.

Anterior TMJ dislocations were found to be 1.3 times more likely to be associated with absence of molars than posterior TMJ dislocations. The probable reasons for this are not clear but it may be due to anterior posturing of the mandible in patients with edentulous posterior segments. Posterior TMJ dislocations have also been associated with posterior edentulousness (30). Anatomical aberrations, as predisposing factors, were not a significant finding in this research.

The cases of TMJ dislocation that were managed were primarily chronic in nature. The probable reasons for this include financial constraints, ignorance, distance from specialised healthcare facilities and mismanagement secondary to the shortage of dental surgeons. This could also be explained by the fact that acute cases were more likely managed at the neighbouring $\mathrm{KNH}$ that is equipped with an accident and emergency centre; while UNDH being a teaching hospital mainly manages specific referral cases and it is not equipped with an accident and emergency centre.

There is mixed opinion regarding the ideal modality of management of TMJ dislocations (30). In the current study it was found that $51 \%$ of the cases were managed conservatively with manual reduction under general anaesthesia being the only conservative modality employed, with a $53 \%$ success rate. Other conservative modalities were not attempted. Hayward points out that conservative methods may often succeed in cases of prolonged dislocation of the TMJ (27). However, according to Shorey and Campbell, in many cases conservative methods promote some temporary relief of symptoms and recurrence is common. Surgical interventions are normally more effective for definite treatment (23). Studies by Marco and Simon (25) show that the most common surgical modalities in the management of TMJ dislocations are the eminectomy and dautrey down-fracturing techniques. In the currentstudy surgical management consisted of two main techniques, eminectomy and condylectomy; eminectomy had a $75 \%$ success rate while condylectomy had a $40 \%$ success rate. Other studies $(23,24)$ have found that among all the different treatment modalities reviewed in literature, a rate of $95 \%$ of cases without recurrence was noted both after eminectomy and use of metallic implant over the articular eminence. In the current study poor follow-up and record keeping limited a detailed analysis of the complications and success of the therapeutic modalities instituted. 
In conclusion, the current study shows that the highest incidence of TMJ dislocation occurs in individuals in the $3^{\text {rd }}$ to $5^{\text {th }}$ decade with a female preponderance. Bilateral anterior TMJ dislocation was found to be the most commont form of dislocation with yawning being the most common aetiology. Most for the cases were managed surgically with eminectomy being the preferred technique with the highest success rate. However, attempts should be made to institute more conservative modalities prior to surgical management. Other surgical techniques, such as the dautrey down-fracture technique and eminence augmentation using metallic implants, should also beinstituted as treatment options. All this, in addition to long-term patient follow-up and good record keeping, will allow for a greater assessment of treatment outcomes and complications.

\section{ACKNOWLEDGEMENTS}

We would like to express our appreciation to the surgeons whose patients were included in the study sample and to the theatre and nursing staff who contributed to the care of these patients. We would also like to thank the anaesthesiologists who actively participated in the operations and general patient care. Finally, wearegrateful totheadministration of theSchool ofDentalSciences, University of Nairobi, for permission to undertake this study and to publish the data.

\section{REFERENCES}

1. Rowe, N.L. and Killey, H.E. Fractures of the facial skeleton 2nd edition; Livingstone Ltd, Edinburgh. 1970; PP 20-35.

2. Lovely, F.W. and Copeland, R.A. Reduction eminoplasty for chronic recurrent luxation of the temporomandibular joint. J. Can. Dent. Assoc. 1981; 47: $179-184$.

3. Smally, A.J. and DelGross, C.Spontaneous dislocation in an 80 year old Man. J. Fam. Prac. 1995; 2: 123-125.

4. Myrhaug, H. Anew method of operation for habitual dislocation of the mandible. Acta. Odont. Scand. 1951; 9: 247-261.

5. Kobayashi, H., Yamazaki, T. and Okudera, H. Correction of recurrent dislocation of the mandible in elderly patients by the Dautrey procedure. $\mathrm{Br}$. J. Oral. \& Maxillofac Surg. 2000; 38: 54-57.

6. Moore, A.P. and Wood, G.D. Medical treatment of recurrent TMJ dislocation using Botulinium toxin A. Br. Dent. J. 1997; 183: 415-417.

7. Adekeye, E.O., Shamia, R.I. and Cove, P. Inverted L-shaped ramus osteotomy for prolonged bilateral dislocation of the TMJ. Oral. Surg. Oral. Med. Oral. Pathol. 1976; 41: 568-577.

8. Chin, R.S., Gropp, H. and Beirne, O.R. Longstanding mandibular dislocation: Report of a case. J. Oral Maxillofac Surg. 1988; 46: 663-696.

9. Kummoona, R. Surgical reconstruction of the TMJ for chronic subluxation and dislocation. Int. J. Oral. Maxillofac Surg. 2001; 30: 344-348.

10. Newton, E., Christian, D.M. and Phil, M. Dislocation, Mandible, emedicine 2004. www.emedicine.com/ emergency/topic147HTM.
11. American Academy of Pediatric Dentistry. Treatment of temporomandibular disorders in children. J. Am. Dent. Assoc. 1990; 120: 265-269.

12. Knibble,A.,Carter,J.B.andFrokjer,G.M.Postanaesthetic temporomandibular joint dysfunction. Anesth. Prog. 1989; 36: 21-25.

13. Rastogi, N.K. and Vakharia, N. Hung operating room: Perioperative anterior dislocation of the Temporomandibular joint. Anaesth. Analg. 1997; 84: 924-926.

14. Mooe, C.A. and Ellis, B. Dislocation of the mandible during the course of cataract surgery. Ophthalmic Surg. 1998; 29: 251-252.

15. Sossis, M. and Lazar, S. Jaw dislocation during general anaesthesia. Can. J. Anaesth. 1987; 34: 407-408.

16. Stella, P.M. Acute infection of the pharynx and tonsils. In Scott Brown's Otolaryngology, 5th edition. Butterworth, London. 1987; 74-118.

17. Maini S., Osbourne J.E., Fadl H. M. S. and Hill, P. TMJ dysfunction following tonsillectomy. Clin. Otolary. Appl. Sci. 2002; 27: 57-58.

18. Collins, D.R., Hogan, J. and Desmond, O. TMJ dislocation associated with stroke. Irish Med. J. 1999; 92: 213-215.

19. Johnson, W.B. New method for reduction of acute dislocation of temporomandibular articulations. J. Oral Surg. 1958; 16: 501-504.

20. Awang, M.N. A new approach to the reduction of acute dislocation of the temporomandibular joint. J. Oral Surg. 1987; 25: 244 -249.

21. Boering, G. Temporomandibular joint osteoartbrosis. A clinical and radiologic investigation. An analysis of 400 cases. Reprints in English of. Arthrosis deformans van het kaakgewricht; een klinische en roen1genologisch onderzoek. Thesis Groningen: Drukkenj van Denderen 1966/1994; 259-267.

22. Schultz, L.W. Report often years experience in treating hypennobility of the TMJ. J. Oral. Surg. 1947; 5: 20022007.

23. Shorey, C.W. and Campbell, J.R. Dislocation of the temporomandibular joint. Oral. Surg. Oral Med. Oral Patho. Oral. Radio Endod. 2000; 89: 662-668.

24. Marco, F. C. and Simon, W. Chronic mandibular dislocation. The role of surgical and non-surgical treatment. J. Can. Dent. Assoc. 1998; 64: 484-491.

25. Daelen, B., Koch, A. and Thorwirth, V. Botulinum toxin treatment of neurogenic dislocation of the temporomandibular joint. Mund. Kiefer. Gosichtschir. 1998; 2: 125-129.

26. Rowe, F. and Caldwell, J. B. Correction of permanent temporomandibular joint dislocation. J. Oral Surg. 1970; 28: 222-226.

27. Hayward, J.R. Prolonged dislocation of the mandible. J. Oral. Surg. 1965; 23: 585-594.

28. Fordyce, G.L. Long-standing bilateral dislocation of the jaw. Br. J. Oral. Surg. 1965; 2: 222-225.

29. Gottleib, J. Long-standing dislocation of the jaw. J. Oral. Surg. 1952; 10: 25-32.

30. Ihalainen U. and Tasanen, A. Central luxation or dislocatinn of the mandibular condyle into the middle cranial fossa. Intern. J. Oral Surg. 1983; 12: 39-45.

31. Alvaro, B. C., Belmiro, C. E. and Oliveira, D.M. Comparative study of eminectomy and use of bone miniplate in the articular eminence for the treatment of recurrent TMJ dislocation. Revista Brasileira de Otorrinolaringologia. 2005; 71: 32-37. 